\title{
Monolithic implementation of AlN-on-silicon bandpass filters with a high-Q notch within the passband
}

\author{
Vikram Thakar and Mina Rais-Zadeh \\ EECS, University of Michigan \\ Ann Arbor, USA \\ thakar@umich.edu
}

\author{
Wanling Pan \\ Integrated Device Technology, \\ San Jose, CA, USA
}

\author{
Farrokh Ayazi \\ School of Electrical Engineering \\ Georgia Institute of Technology \\ Atlanta, USA
}

\begin{abstract}
In this paper, we present acoustically coupled filters capable of inducing a high- $Q$ notch within the filter passband. The origin of the notch is a result of charge cancellation across the output electrodes, which is a direct consequence of exciting higher order lateral modes with a displacement profile out-ofphase with the primary modes of the filter. We show multiple filters with a passband notch and demonstrate the viability of using top electrode layout to adjust the placement of the notch within the filter passband. A modified equivalent circuit is used to intuitively explain the response of a bandpass filter with an integrated notch. Such filters can have applications as pre-select bandpass filters capable of rejecting in-band interfering signals.
\end{abstract}

\section{INTRODUCTION}

Over the past decade, wireless communication has enabled an increasing number of applications, many of which, such as wireless sensor nodes and implanted bio-sensors, have been made possible because of the progress made in miniaturization of the wireless systems [1]. While such systems have had a significant positive impact, they have also resulted in the problem of spectrum crowding. Considering limitations in the available spectrum, a major area for research is in optimizing the use of existing spectrum allocations [2].

Communication modules rely on passive filters at the front end in order to improve the signal to noise ratio and select the band of interest. Acoustically coupled piezoelectric filters have been proposed as an attractive alternative in such applications [3], [4]. A commonly used implementation for such filters is the thin film piezoelectric on substrate (TPoS) platform. In such filters, the input and output electrodes are coupled via evanescent waves in the unelectroded regions to generate a passband response. A typical filter shows a low insertion loss $(<3 \mathrm{~dB})$ with a bandwidth that can be designed to be within 0.1 to $1 \%$ of the center frequency [5].

In this paper, we present a solution to implement acoustically coupled filters with a high- $Q$ notch within the passband. The notch can be used to eliminate any strong interfering signals at specific frequencies within the communication band.

This work was partially supported by Integrated Device Technology (IDT) Inc., NSF (Award \# 1055308), and University of Michigan.
The device presented is modeled both analytically and using finite element methods to explain the origin of the notch. From the presented analysis, it will be shown that the notch is a result of charge cancellation across the output electrodes caused due to the excitation of higher order lateral modes in the structure. The frequency characteristics of the notch are shown to be directly dependent on the $Q$ of this high-order mode and its effective coupling. From measurements across the wafer, we show multiple devices with varying notch position indicating the possibility to reject interference signals at targeted frequencies.

\section{WORKING PRINCIPLE AND DEVICE FABRICATION}

Figure 1 shows the structure of a typical TPoS acoustic filter. In this structure, two or more resonating electroded sections of the stack are coupled to one another via the unelectroded regions. The top electrode consists of two interdigitated transducers (input and output) and the bottom electrode is a solid metal layer that is connected to the electrical ground.

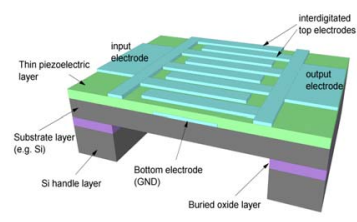

(a)

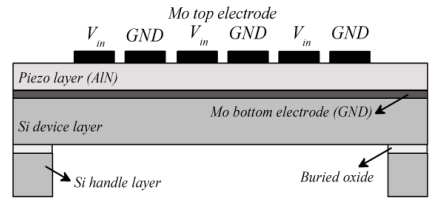

(b)
Figure 1: (a) Structure of a TPoS filter. (b) 2D model used in finite element analysis.

The working principle of such acoustically coupled filters can be intuitively explained using the dispersive properties of lamb waves in the structure. Using Matrix techniques, the dispersion curves for the thickness extensional (TE) mode can be calculated. Fig. 2 plots the dispersion curve of an exemplary stack with the dimensions specified in the fabrication flow in Fig. 3 [6]. The two curves (in red) are the TE mode dispersion relations in the electroded and unelectroded regions of the filter, respectively. These curves represent the supported lateral wavenumbers (or wavelengths) at a given input excitation frequency. Within the cut-off 
region, for travelling waves in the electroded region, the unelectroded region will support evanescent waves leading to lateral modes in the system [7]. A multitude of such lateral modes contribute to form the filter bandpass response. The measured response of a notched bandpass filter (blue) is overlaid in Fig. 2 for ease of comparison. The process flow used for fabricating these devices is summarized in Fig. 3 [3].

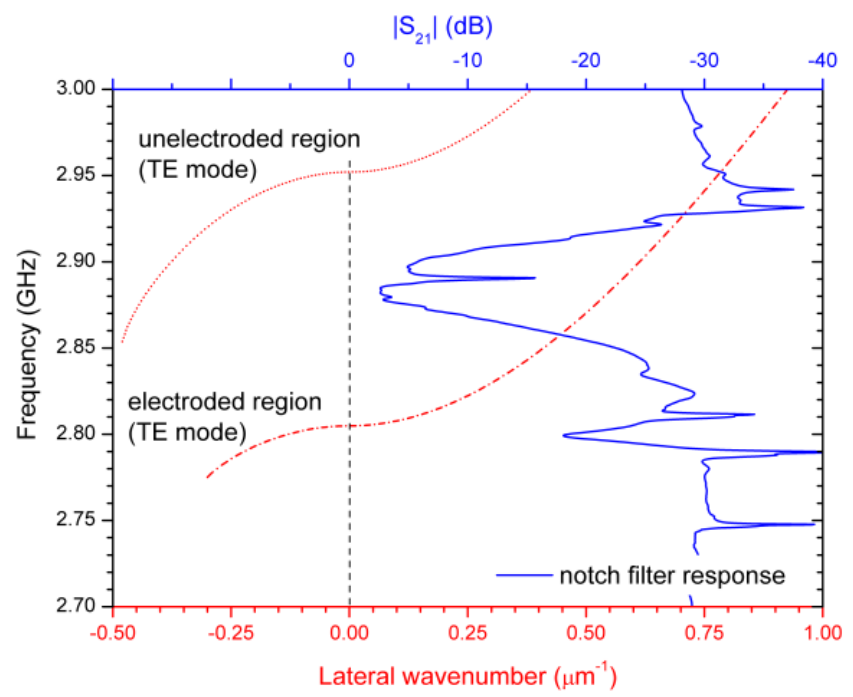

Figure 2: Calculated dispersion curves for the thickness extensional (TE) mode for the filter stack used in this work. The measured filter response is overlaid for comparison (electrode width $=10 \mu \mathrm{m}$, spacing $=3 \mu \mathrm{m}$, and number of electrode fingers $=40$ ).

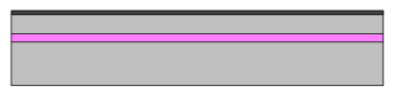

(a) SOI wafer (device layer $5 \mu \mathrm{m}$ ). Sputter Mo as bottom metal

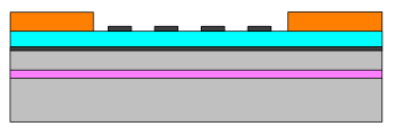

(c) Sputter Mo as the top electrode. Evaporate thick Ti/Au pads.

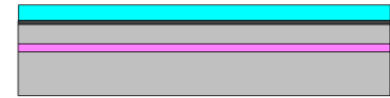

(b) Sputter $1 \mu \mathrm{m}$ AIN as the transduction layer

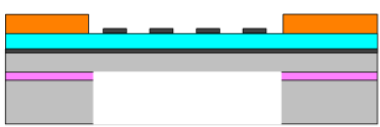

(d) DRIE to release structure. Remove BOX layer using RIE.
Figure 3: Fabrication process flow used for the filters shown in this work. Both top and bottom electrodes are $100 \mathrm{~nm}$ thick.

\section{MEASUREMENT RESULTS AND MODELNG}

Figure 4 plots the measured response of two filters with the same filter stack: $5 \mu \mathrm{m}$ Silicon, $100 \mathrm{~nm} \mathrm{Mo,} 1 \mu \mathrm{m}$ AlN and $100 \mathrm{~nm} \mathrm{Mo}$, but with different top electrode layout. The spacing between the top electrodes of the filter in Fig. 4(a) is $5 \mu \mathrm{m}$, while that of the filter in Fig. 4(b) is $3 \mu \mathrm{m}$. Both filters have 40 electrode fingers each $10 \mu \mathrm{m}$ wide.

The frequency response of a TPoS filter as seen in this work can be simulated with great accuracy using finite element methods [5]. The fitted simulation result along with the measured result is plotted in Fig. 5. It should be noted here that in a previous work [5], simulations with this design geometry $(10 \mu \mathrm{m}$ electrode width and $3 \mu \mathrm{m}$ finger spacing) did not show an integrated notch in the passband. This discrepancy can be attributed to geometric variations seen in the fabricated devices. To explain the presence of the notch for the filter in Fig. 4 (b), the device was simulated using the detailed procedure outlined in [5]. The actual geometry and thickness of the materials in the filter stack were measured using a scanning electron microscope (SEM) and the frequency response is fitted by allowing small variations in the measured geometry. As an example, the simulation fit required the finger spacing to be $3.15 \mu \mathrm{m}$ as compared to the design value of $3 \mu \mathrm{m}$.
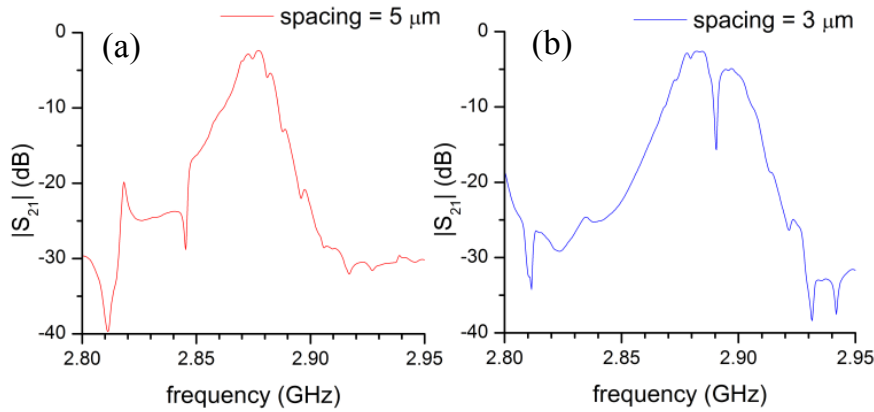

Figure 4: Measured response of a filter with (a) $5 \mu \mathrm{m}$ and (b) $3 \mu \mathrm{m}$ finger spacing. Both filters have the same stack, electrode width of $10 \mu \mathrm{m}$ and finger length of $200 \mu \mathrm{m}$. Number of fingers is 40 in each case.

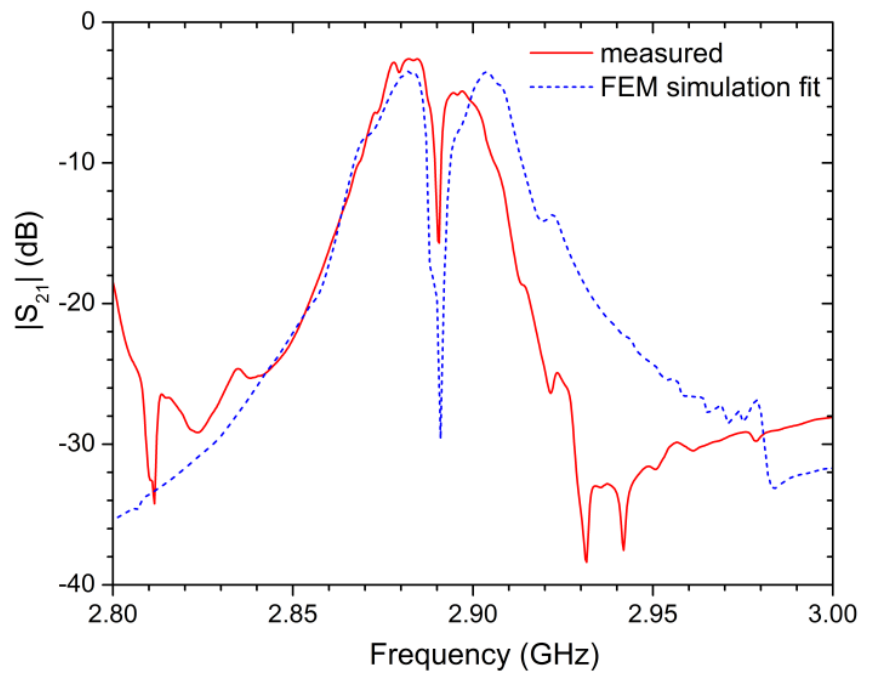

Figure 5: Simulated frequency response from finite element analysis using COMSOL is overlaid along with the measured filter response for ease of comparison. The top electrode geometry consists of 40 electrode fingers each $10 \mu \mathrm{m}$ wide with a spacing of $3 \mu \mathrm{m}$ (design parameters).

These results illuminate certain characteristics of the thickness mode filters. First, the same stack can be used to build filters with and without an integrated notch within the passband by only changing the lateral electrode geometry. Second, a well-controlled process is essential to achieve a repeatable performance but such a process would allow a great number of filter variations in a highly integrated platform.

While finite element modeling provides a good fit to the measured results, explaining the origin of the notch from the results is much more challenging. To analyze the origin of the notch, we choose to implement an analytical model presented recently [7]. Using this model, the lateral modes of the system can be explicitly calculated and the frequency response can be constructed using their modal contributions. Using the frequency-wavenumber data (as shown in Fig. 2) and following the work presented in [7], the lateral modes supported by the filter geometry are calculated and the 
dominant in-phase and out-of-phase modes are presented in Fig. 6.
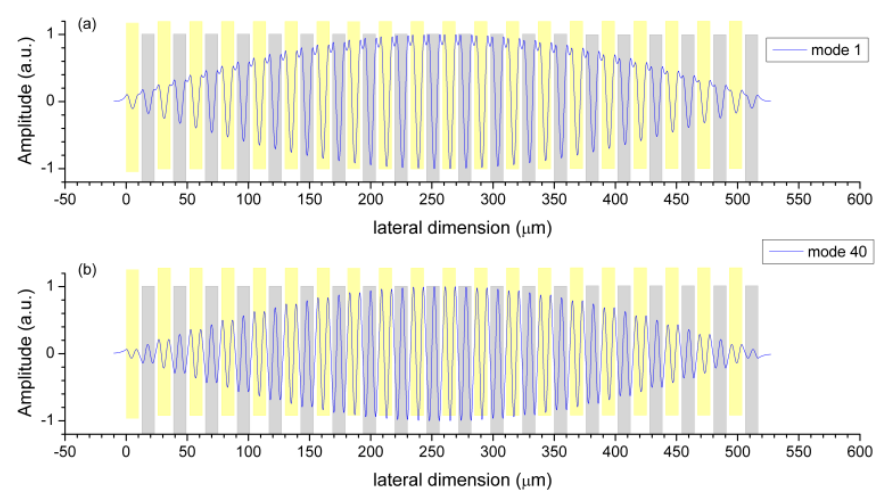

Figure 6: First order lateral (a) in-phase and (b) out-of-phase modes of the filter. The $y$-axis plots the normalized displacement perpendicular to the plane of the electrodes across the device width. The input (light yellow) and output (light grey) electrodes are shown for clarity.

From the model for lateral coupling, the complete set of lateral modes contributing towards the system response can be calculated. The modes for the device under consideration are calculated and plotted in Appendix I. The calculated coupling of each mode, as defined in [7], normalized to the coupling of mode 40 is listed in Table 2, Appendix I. The frequency response constructed from the calculated lateral modes provides great insight into the effect of each individual mode on the overall response. Based on this analysis, we can deduce that the $9^{\text {th }}$ harmonic of the lateral in-phase mode (mode 9) creates a sharp notch for the case of 40 electrode fingers. The calculated frequency response and the $9^{\text {th }}$ harmonic mode are plotted in Fig. 7.
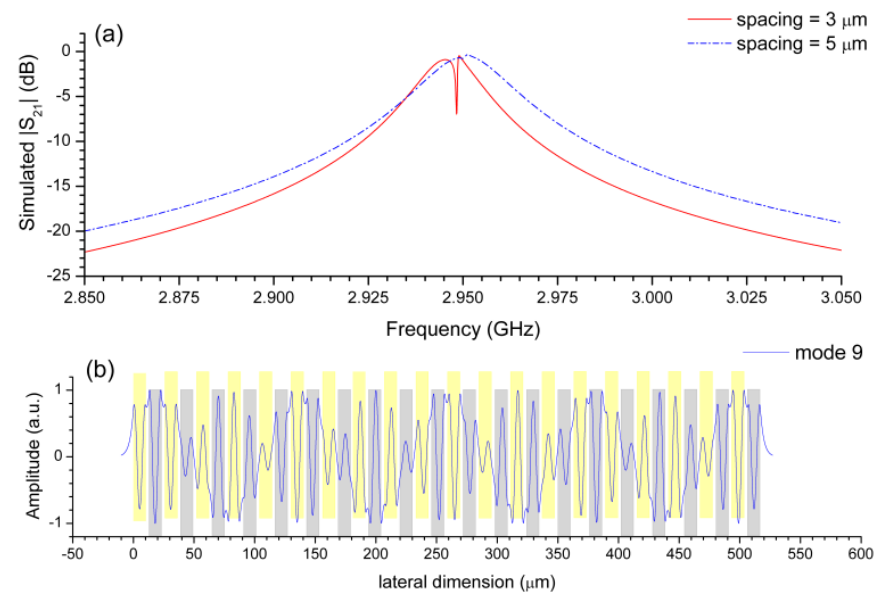

Figure 7: (a) Calculated frequency response of a TPoS filter having 40 electrode fingers each $10 \mu \mathrm{m}$ wide and having a spacing of $3 \mu \mathrm{m}$ and $5 \mu \mathrm{m}$ respectively. The $Q$ of mode 9 is taken to be 20,000 while that of the dominant in- phase mode (mode 1) is taken to be 2,000. (b) Mode shape for the $9^{\text {th }}$ harmonic of the lateral in-phase mode (mode 9). The input (light yellow) and output (light grey) electrodes are shown for clarity.

In this case, it was observed that the $9^{\text {th }}$ harmonic mode leads to a sharp notch when its $Q$ is at least 10 times higher than the $Q$ of the primary in-phase mode (Fig. 6(a)). From the measured response one can see that the notch has a very high $Q$ and thus, there is an agreement between calculations and measured results.
Figure 8 shows the total calculated displacement profile across the electrodes at the notch frequency (refer Fig. 7 (a)). At this frequency, the average displacement across the output electrodes is very small indicating that the notch is a result of charge cancellation across the output electrodes.

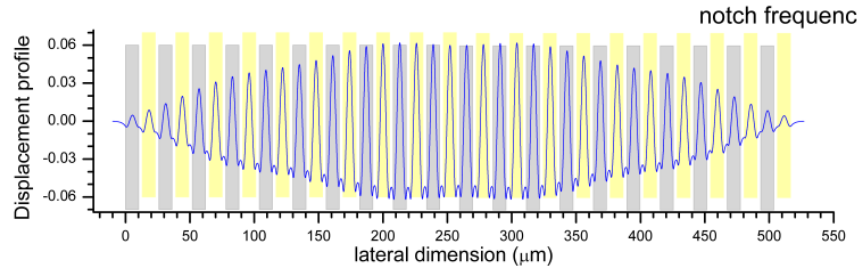

Figure 8: Calculated displacement profile across the electrodes (shaded light yellow for the output and light grey for the input) at the frequency of notch (refer notch in Fig. 7(a)). The average displacement across the output electrodes is very small leading to a notch formation within the passband.

From the analysis, it is seen that the number of lateral modes (only for the TE mode of the plate) generally equals the number of electrode fingers used in the filter (See appendix I). Thus by increasing the number of electrode fingers an increased number of modes can be excited and as a consequence, we have an increased number of modes suitable of inducing a notch within the passband. As an example, modes 3, 5, 7, 9 and 11 are all capable of inducing a notch in the filter passband in the example shown in Fig. 7. The choice of mode 9 comes from the position of the notch (measured) within the passband. In case of the filter with 20 electrodes (Fig 9), the $5^{\text {th }}$ lateral overtone is responsible for causing the notch. This implies possibilities of changing the frequency of the notch by exciting modes of different order that result in charge cancellation across the output electrodes. This hypothesis is supported by the measured results of filters with increasing number of fingers as shown in Fig. 9. A possible extension of this concept would be to excite multiple high- $Q$ lateral overtones to incorporate multiple notches within the filter passband.
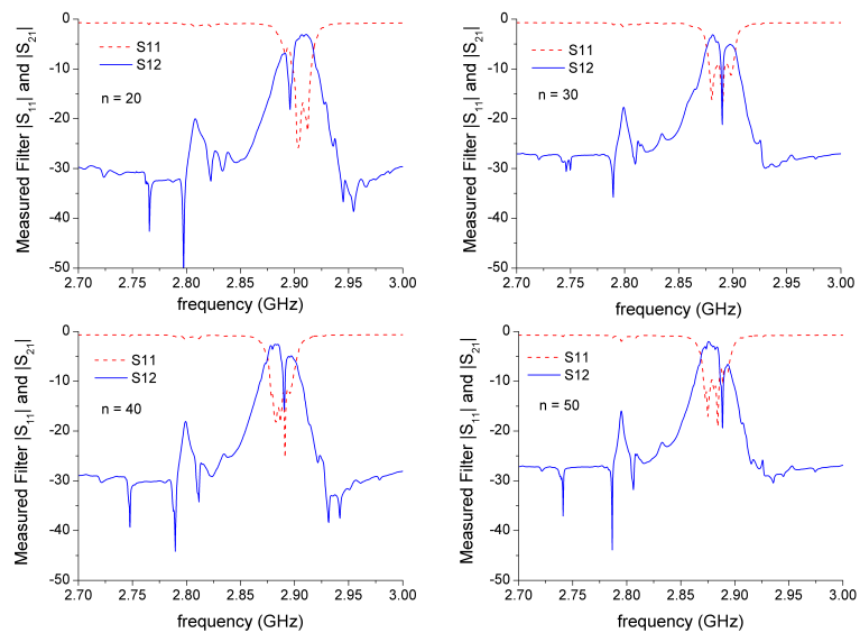

Figure 9: Measured response for filters demonstrating a shift in the notch frequency. The number of electrodes in each case is marked on the figure. Results indicate the dependence of the notch position on the top electrode geometry. Increasing number of fingers changes the lateral overtone mode having a high $Q$, thus changing the position of the notch. 


\section{EQUIVALENT CIRCUIT}

The notched filter is also modeled using an equivalent electrical circuit shown in Fig. 10.

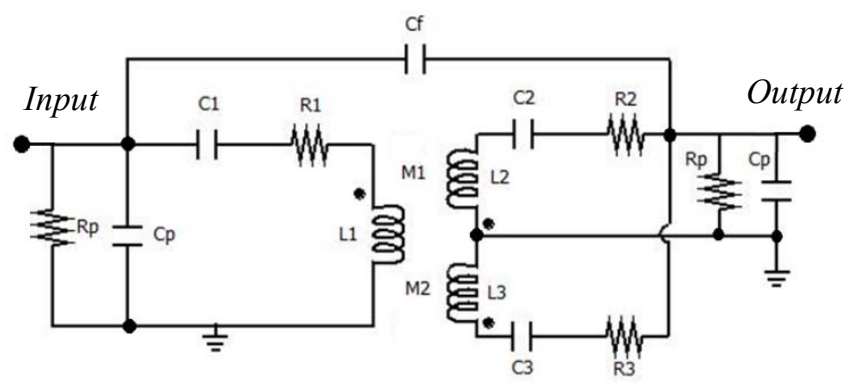

Figure 10: Modified equivalent electrical circuit used to model laterally coupled filters with a notch.

The equivalent circuit model used here is a modification of the standard implementations used in previous works [3], [4]. It allows for an intuitive explanation of the filter working principle. The energy coming into the system from the input electrodes is considered to be coupled to the output via the inphase and the out-of-phase modes. The ratio of the energy coupled from the input to the output is set by the transformer ratios. While this model provides good insight into the filter transfer function, one can easily deduce that in this case $\mathrm{S}_{11} \neq \mathrm{S}_{22}$, which is essential for passive and symmetric structures. A further modification providing two resonators at the input, similar to the structure at the output can be implemented to make the circuit balanced. Figure 11 plots the fitted circuit response overlaid with the measured response for comparison. The fitted circuit parameters are tabulated in Table 1.

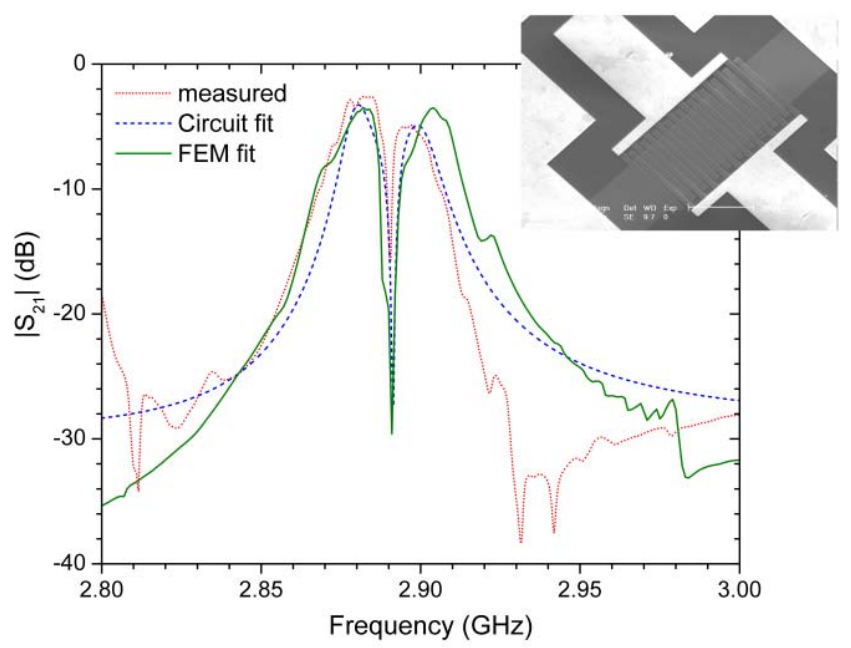

Figure 11: The circuit fit obtained using the equivalent electrical circuit is overlaid along with the measured filter response and the FEM fit (obtained earlier) for ease of comparison. The top electrode geometry consists of 40 electrode fingers each $10 \mu \mathrm{m}$ wide with a spacing of $3 \mu \mathrm{m}$ (design parameters). Inset shows the SEM image of a typical device with 20 fingers.
Table 1: Circuit parameters used to fit the measured response

\begin{tabular}{|c|c|c|c|c|c|}
\hline Label & Value & Label & Value & Label & Value \\
\hline $\mathrm{R}_{1}$ & $5 \Omega$ & $\mathrm{C}_{1}$ & $11.07 \mathrm{fF}$ & $\mathrm{L}_{1}$ & $274.4 \mathrm{nH}$ \\
\hline $\mathrm{R}_{2}$ & $3 \Omega$ & $\mathrm{C}_{2}$ & $11.07 \mathrm{fF}$ & $\mathrm{L}_{2}$ & $275.9 \mathrm{nH}$ \\
\hline $\mathrm{R}_{3}$ & $5 \Omega$ & $\mathrm{C}_{3}$ & $2.03 \mathrm{fF}$ & $\mathrm{L}_{3}$ & $1494.5 \mathrm{nH}$ \\
\hline $\mathrm{R}_{\mathrm{p}}$ & $450 \Omega$ & $\mathrm{C}_{\mathrm{p}}$ & $1.425 \mathrm{pF}$ & $\mathrm{M}_{1}$ & $-1.7 \mathrm{nH}$ \\
\hline & & $\mathrm{C}_{\mathrm{f}}$ & $58 \mathrm{fF}$ & $\mathrm{M}_{2}$ & $+1.4 \mathrm{nH}$ \\
\hline
\end{tabular}

\section{V.CONCLUSION}

In this paper we demonstrated a TPoS implementation of a passband filter that exhibits an integrated notch within the passband. The origin of the notch is identified as a high- $Q$ overtone of the in-phase lateral mode. The design of bandpass filters integrated with a high-rejection notch would thus entail designing the $Q$ of a specific lateral mode to be high. While finite elements are shown to provide good prediction of notch characteristics, reliable design of filters with a high $Q$ integrated notch will require improved analytical models capable of calculating modal quality factors.

The position of the notch was shown to have a dependence on the number of electrode fingers through selection of the lateral overtone having a high $Q$. Such mode selectivity by means of only geometric variations could have great potential in filter implementations for various applications.

\section{REFERENCES}

[1] I.F. Akyildiz, W. Su, Y. Sankarasubramaniam, and E. Cayirci, "Wireless sensor networks: a survey," Computer Networks, vol. 38, issue 4, pp. 393-422, March 2002.

[2] P. Leaves, et al., "Dynamic spectrum allocation in composite reconfigurable wireless networks," Communications Magazine, IEEE, vol. 42, no. 5, pp. 72-81, May 2004.

[3] W. Pan, V. Thakar, M. Rais-Zadeh and F. Ayazi, "Acoustically coupled thickness-mode AlN-on-Si bandpass filters Part I : Principle and Devices," IEEE Trans. Ultrason. Ferroelectr. Freq. Control (unpublished).

[4] W. Pan, R. Abdolvand and F. Ayazi, "A low-loss $1.8 \mathrm{GHz}$ monolithic thin-film piezoelectric-on-substrate filter," IEEE MEMS, pp. 176-179, Tucson, Arizona, Jan. 2008.

[5] V. Thakar, W. Pan, F. Ayazi and M. Rais-Zadeh, "Acoustically coupled thickness-mode AlN-on-Si bandpass filters Part II : Simulation and Analysis," IEEE Trans. Ultrason. Ferroelectr. Freq. Control (unpublished).

[6] M. Lowe, "Matrix techniques for modeling ultrasonic waves in multilayered media," IEEE Trans. Ultrason. Ferroelectr. Freq. Control, vol. 42, no. 4, pp. 525-541, 1995.

[7] T. Pensala, J. Meltaus, K. Kokkonen and M. Ylilammi, "2-D modeling of laterally acoustically coupled thin film bulk acoustic wave resonator filters," IEEE Trans. Ultrason. Ferroelectr. Freq. Control, vol. 57, no. 11, pp. 2537-2549, Nov. 2010. 


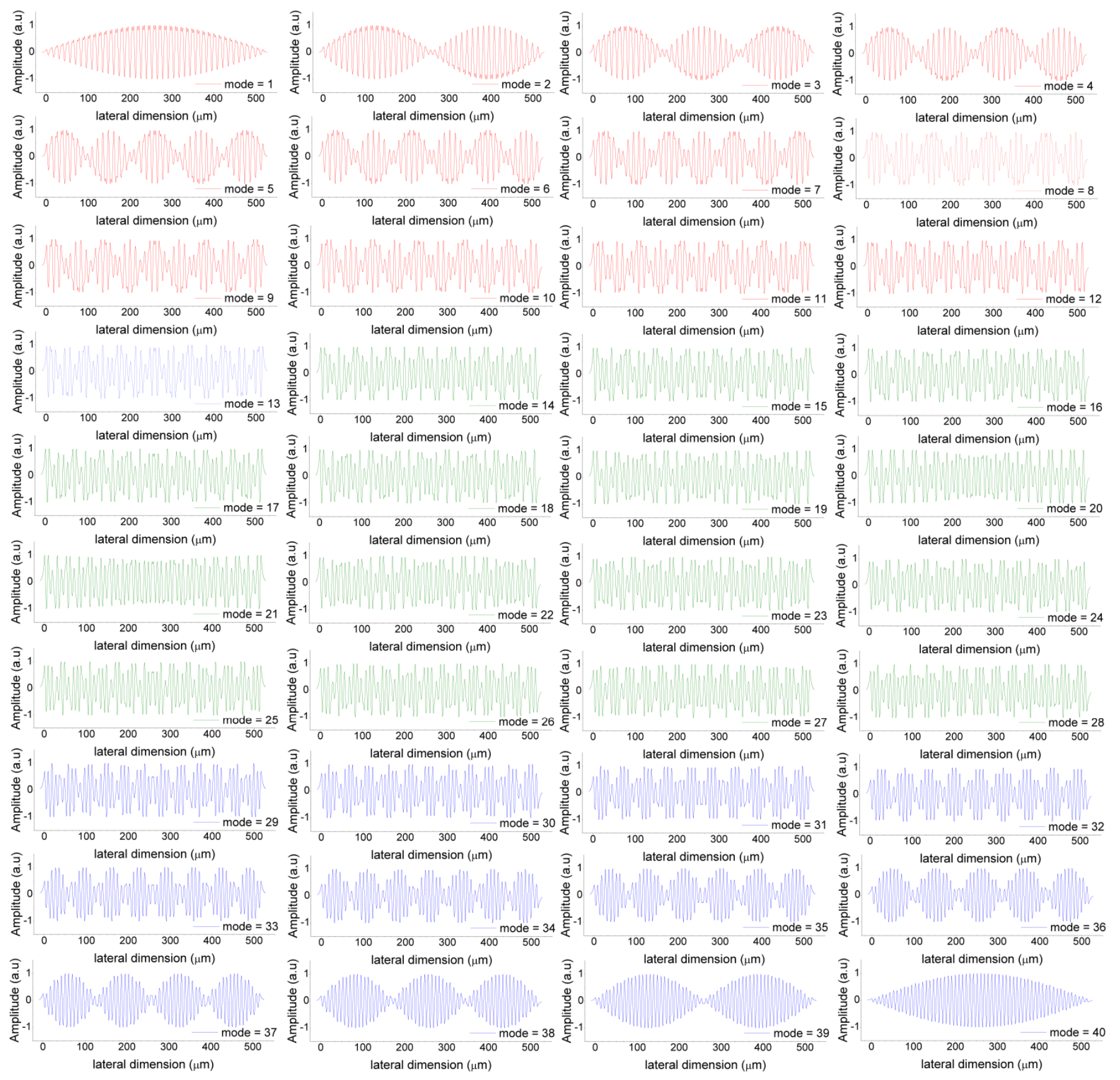

Figure 12: Calculated lateral modes for a TPoS filter with 40 electrode fingers. Each finger is $10 \mu \mathrm{m}$ wide and the spacing between them is $3 \mu \mathrm{m}$. Modes $1-12$ (in red) are the prominent in-phase lateral modes while modes 29-40 (in blue) show a prominent out-of-phase response.

Table 2: Calculated coupling values for the modes shown in Fig. 12, normalized to the coupling of mode 40 . The coupling of each mode is calculated by taking the ratio of the integral of displacement across the output electrodes to the total energy of the mode [7]

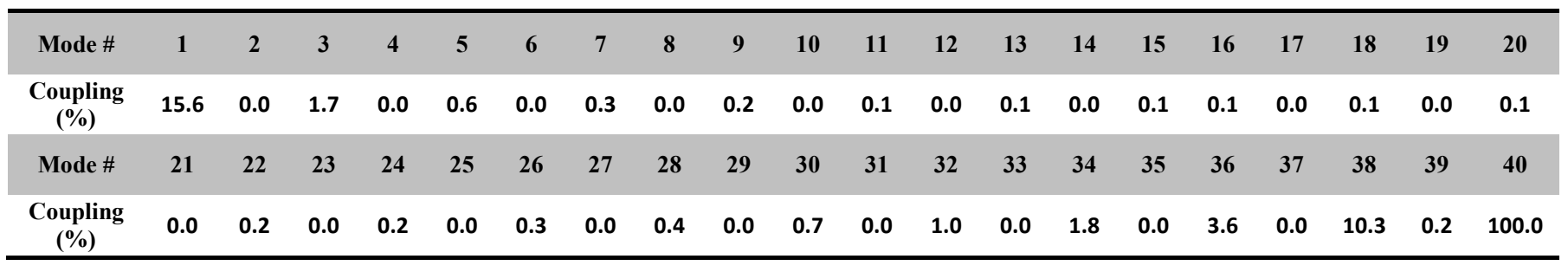

